\title{
GLP-1 receptor agonists vs. SGLT-2 inhibitors: the gap seems to be leveling off
}

\author{
Dario Giugliano $0^{1,2^{*}} \mathbb{0}$, Lorenzo Scappaticcio ${ }^{1,2}$, Miriam Longo ${ }^{1}$, Giuseppe Bellastella ${ }^{1,2}$ and Katherine Esposito ${ }^{2,3}$
}

\begin{abstract}
Cardiovascular disease (CVD) remains the leading cause of death in patients with type 2 diabetes (T2D). Older age, prior heart failure (HF) and CV events, peripheral artery disease, and kidney complications can identify a subgroup of patients with T2D at high risk of mortality who are likely to achieve the greatest benefit from newer glucose-lowering agents. Both glucagon-like peptide-1 receptor agonists (GLP-1RA) and sodium-glucose cotransporter-2 (SGLT-2) inhibitors can reduce $\mathrm{CV}$ risk in patients with T2D, and both are recommended by the American Diabetes Association to reduce the risk of major cardiovascular events (MACE). The magnitude of the benefits of GLP-1RA and SGLT-2 inhibitors on MACE are similar, ranging from 12 to 14\% reduction of risk, but only GLP-1RA may reduce the risk of stroke. The most striking difference between the two classes of drugs relates to the amelioration on hospitalization for HF, as the benefit of SGLT-2 inhibitors surpass by threefold that obtained with GLP-1RA. Despite this, GLP-1RA also exert a significant benefit on HF which suggest their use when SGLT-2 inhibitors are contraindicated or not tolerated. The difference between the two classes is less impressive for the kidney outcome. Overall, the results of CVOTs published so far seems to suggest that the gap between the cardiorenal benefits of SGLT-2 and GLP-1RA is narrowing.
\end{abstract}

Keywords: Type 2 diabetes, SGLT-2 inhibitors, GLP-1 receptor agonists, Cardiorenal benefits

In 1999, the American Heart Association stated that "diabetes is a cardiovascular disease" [1]. A review of 2018 including data from 57 articles involving 4 million people indicated that the overall prevalence of cardiovascular disease (CVD) in diabetic patients was 32.2\% [2]. Still today, individuals with diabetes have an approximately two-fold increased risk of all-cause mortality than those without diabetes [3]. On the other hand, the cardiovascular destiny of the diabetic patient is not unavoidable, as patients with type 2 diabetes (T2D) who had major risk factors for cardiovascular disease (CVD) within the target range had little or no excess risk of CVD and mortality [4]. Unfortunately, only $5-6 \%$ of people with T2D had optimal risk factor control $[4,5]$. In a contemporary

\footnotetext{
*Correspondence: dario.giugliano@unicampania.it

${ }^{1}$ Division of Endocrinology and Metabolic Diseases, Department

of Advanced Medical and Surgical Sciences, University of Campania Luigi Vanvitelli, Naples, Italy

Full list of author information is available at the end of the article
}

cohort of 16,492 patients with T2D and at high/very high CV risk participating in the SAVOR-TIMI 53 trial [6], CVD remained the leading cause of death and approximately one-third of all deaths were classified as sudden. Older age, prior heart failure (HF) and CV events, peripheral artery disease, and kidney complications can identify a subgroup of patients with T2D at high risk of mortality who are likely to achieve the greatest benefit from aggressive management of modifiable risk factors and newer glucose-lowering agents.

Two classes of newer anti-hyperglycemic agents can reduce $\mathrm{CV}$ risk and events in patients with $\mathrm{T} 2 \mathrm{D}$, namely glucagon-like peptide-1 receptor agonists (GLP-1RA) and sodium-glucose cotransporter-2 (SGLT-2) inhibitors. GLP-1RA can reduce MACE (major cardiovascular events) and its individual components, CV death, myocardial infarction (MI) and stroke. These beneficial effects of GLP-1RA on MACE are independent of many variable including the presence of established $\mathrm{CV}$ disease at baseline, the structural basis of GLP-1RA (exendin-4 
based agonists vs. human GLP-1- based molecules), the daily or weekly administration of the agonist, baseline HbA1c, body weight, age (> 65 vs. $\leq 65$ years), baseline eGFR ( $<60 \mathrm{vs.} \geq 60 \mathrm{ml} / \mathrm{min}$ per $1.73 \mathrm{~m}^{2}$ ) and duration of follow-up of the trial ( $<3 \mathrm{vs.} \geq 3$ years) [7, 8]. Intuitively, therapy with GLP-1RA can be beneficial in patients with T2D and established CVD or at risk for CVD. Accordingly, the last recommendation of the American Diabetes Association (ADA, Standard of Care 2021) states that in patients with T2D and established CVD or multiple risk factors for CVD, a GLP-1RA with demonstrated cardiovascular benefit is recommended to reduce the risk of MACE [9].

SGLT-2 inhibitors also reduce the risk of atherosclerotic MACE in patients with T2D with or without established CVD [10, 11]. SGLT-2 inhibitors reduce the risk of hospitalization for HF and progression of kidney disease in patients with established CVD, multiple risk factors for CVD, or diabetic kidney disease. According to this evidence, ADA states that in patients with T2D and established CVD, multiple CVD risk factors, or diabetic kidney disease, an SGLT-2 inhibitor with demonstrated cardiovascular benefit is recommended to reduce the risk of MACE and/or hospitalization for HF [9]. Moreover, in patients with T2D and established HF with reduced ejection fraction (HFrEF), an SGLT-2 inhibitor with proven benefit in this patient population is recommended to reduce the risk of worsening $\mathrm{HF}$ and $\mathrm{CV}$ death. The benefits seen in this patient population likely represent a class effect, and they appear unrelated to glucose lowering given comparable outcomes in HFrEF patients with and without diabetes. For many patients, use of either an SGLT-2 inhibitor or a GLP-1RA to reduce CV risk is appropriate. It is unknown whether use of both classes of drugs will provide an additive cardiovascular benefit.

Ongoing trials are assessing the effects of several SGLT-2 inhibitors in patients with HF and reduced or preserved ejection fraction. The results of the EMPEROR-P [12] have shown that in 5988 patients with or without T2D and with HF and preserved ejection fraction $(>40 \%)$, treatment with $10 \mathrm{mg}$ empagliflozin for a median time of 26.2 months reduced the risk of a composite of CV death or hospitalization for HF by $21 \%$, and effect which was mainly related to a $29 \%$ lower risk of hospitalization for HF. The benefit of empagliflozin was independent of the diabetic status. According to the results of both DAPA-HF [13] and EMPEROR-R [14], the FDA have approved both dapagliflozin and empagliflozin to reduce the risk of $\mathrm{CV}$ death or hospitalization for $\mathrm{HF}$ in adults with HF and reduced ejection fraction regardless of whether they have diabetes.

The magnitude of the benefits of GLP-1RA and SGLT-2 inhibitors on MACE are similar in patients with T2D, ranging from 12 to $14 \%$ reduction of risk, but only GLP1RA may reduce the risk of stroke (Fig. 1). The most striking difference between the two classes of drugs relates to the amelioration on hospitalization for HF, as the benefit of SGLT-2 inhibitors surpass by threefold that obtained with GLP-1RA. Despite this, GLP-1RA also exert a significant benefit on HF which suggests their use when SGLT-2 inhibitors are contraindicated or not tolerated. The difference between the two classes is less impressive for the kidney outcome; moreover, similar GLP-1RA effect sizes suggest a lack of sufficient power rather than a lack of effect. Overall, the results of CVOTs published so far seems to suggest that the gap between the cardiorenal benefits of SGLT-2 and GLP-1RA is narrowing.

Previous analyses have suggested a larger benefit of SGLT-2 inhibitors, as compared with GLP-1RA, on cardiorenal events. For instance, a large network meta-analysis showed that SGLT-2 inhibitors reduced hospitalization for HF and renal composite outcome more than GLP-1RA [15], and that in patients with cardiovascular disease, SGLT-2 inhibitors show significant reduction in both heart failure (HF) hospitalizations and mortality for patients with HF and reduced ejection fraction [16].

Moreover, a retrospective real-world study shows that both GLP1-RA and SGLT-2 inhibitors reduce the 10-year risk for cardiovascular disease in patients with T2D in primary cardiovascular prevention [17], although SGLT-2 inhibitors seem to have a greater cardioprotective benefit compared to GLP-1RA when used for secondary prevention among adults with T2D [18].

Prescriptions of the newer anti-hyperglycemic agents continue to stagnate, even among eligible patients [19], which may be related, at least in part, to the uncertainty about the optimal clinical management of T2D. Sources of uncertainty originate from the panoply of glycemic targets, the complexity of drug therapy, the choice of the first drug, the ideal sequence of drugs after the first drug failure, the possible harms of anti-hyperglycemic drugs, the outcomes of treatment (surrogate versus clinical) and the hierarchy of risk factors to treat for preventing the vascular complications. Ironically, multiple treatment guidelines and algorithms periodically released to improve guidance may generate confusion into clinicians [20]. Moreover, treatment algorithms cannot be truly evidence-based because of a lack of studies comparing all available treatment combination options. Confusion likely contributes to clinical inertia $[20,21]$, thereby effectively denying evidence-based treatments advocated to patients with T2D and CVD. Coordinated action is required to ensure that people with type 2 diabetes, cardiovascular disease, heart failure, or chronic kidney disease are treated appropriately 


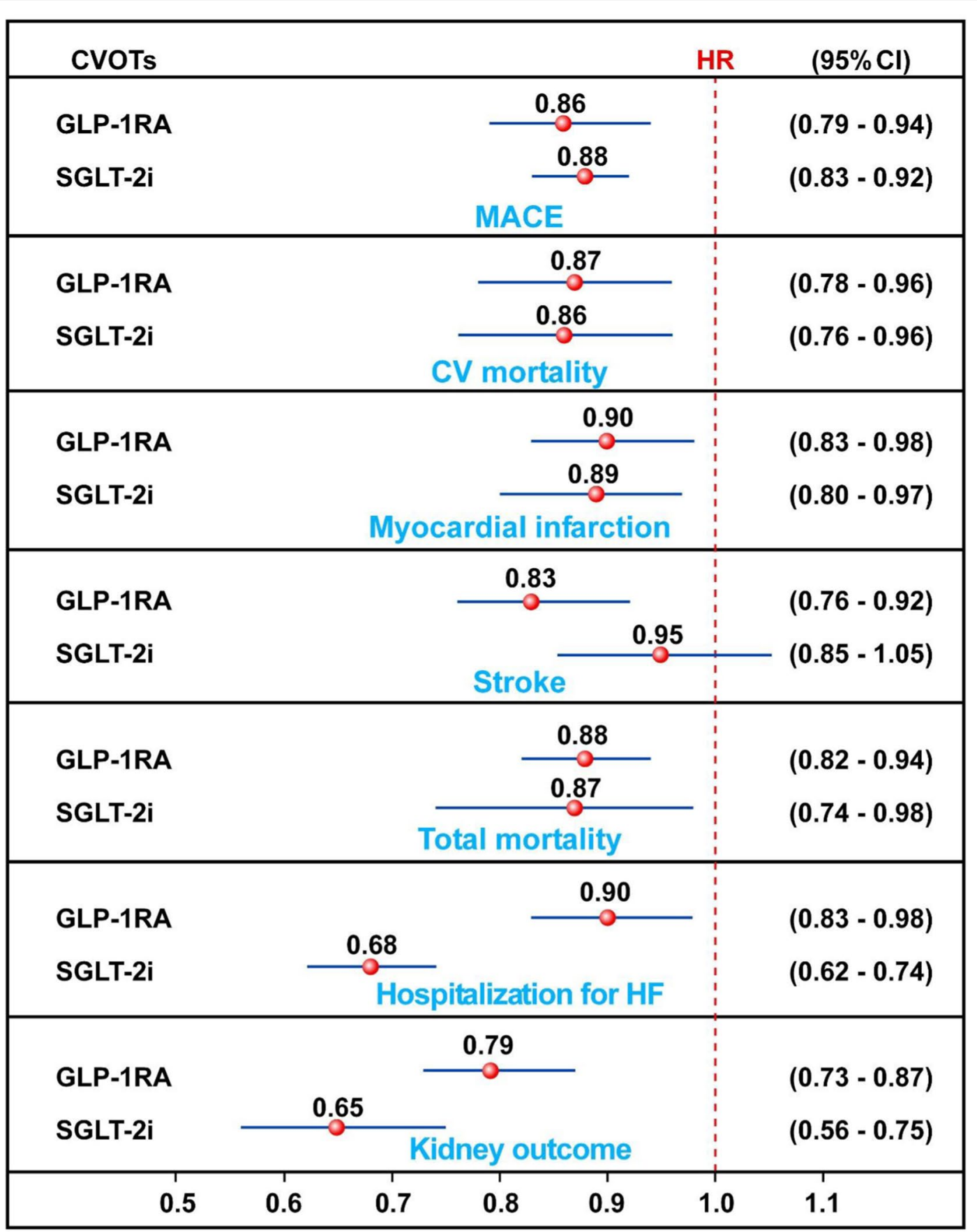

Fig. 1 Meta-analyses of cardiorenal effects exerted by GLP-1RA and SGLT-2 inhibitors in patients with or without type 2 diabetes. HR hazard ratio, $\mathrm{Cl}$ confidence intervals. The results are based on data in Ref. [7, 8, 11]

with an SGLT-2 inhibitor or GLP-1RA. Moreover, more adults with diabetes in the US have suboptimal glycemic control now compared to 10 years ago [22]. In adult NHANES (National Health and Nutrition Examination Survey) participants with diagnosed diabetes, glycemic control declined after more than a decade of progress, associated with a resurgence in vascular diabetic complications [23].

SGLT-2 inhibitors and GLP-1RA represent antihyperglycemic therapies shown to reduce CVD and chronic kidney disease risks in patients with T2D. In addition, SGLT-2 inhibitors have shown benefit in patients with both 
HFrEF and HFpEF independently of diabetes status, which opens exciting possibilities for the use of these therapies in patients at risk for or with established $\mathrm{CV}$ or kidney disease without T2D. There is an urgent need to incorporate multidisciplinary care in the identification of high-risk patients who may benefit from these agents [24]. Finally, legislative support should promote equitable access to these agents, especially for vulnerable and underrepresented patient populations who also carry the highest burden of CVD and CKD risk with T2D.

\begin{abstract}
Abbreviations
CVD: Cardiovascular disease; T2D: Type 2 diabetes; CV: Cardiovascular; HF: Heart failure; GLP-1RA: Glucagon-like peptide 1 receptor agonist; SGLT-2: Sodium-glucose cotransporter 2; MACE: Major cardiovascular events; MI: Myocardial infarction; ADA: American Diabetes Association; HFrEF: Heart failure with reduced ejection fraction; HFpEF: Heart failure with preserved ejection fraction; FDA: Food and Drug Administration; NHANES: National Health and Nutrition Examination Survey; CKD: Chronic kidney disease.
\end{abstract}

\section{Acknowledgements}

Not applicable.

\section{Author's contributions}

DG wrote the manuscript. LS, ML, GB and KE critically reviewed the manuscript. All authors read and approved the final manuscript.

\section{Funding}

No funding

\section{Availability of data and materials}

All data generated or analyzed during this study are included in this published article.

\section{Declarations}

Ethics approval and consent to participate

Not applicable.

\section{Consent for publication}

Not applicable.

\section{Competing interests}

D.G. received honoraria for speaking at meetings from Novartis, Sanofi, Lilly, Astrazeneca, and NovoNordisk. K.E. received honoraria for speaking at meetings from Novartis, Sanofi-Aventis, Lilly, AstraZeneca, Boehringer Ingelheim, and NovoNordisk. No other potential conflict of interest relevant to this article was reported.

\section{Author details}

'Division of Endocrinology and Metabolic Diseases, Department of Advanced Medical and Surgical Sciences, University of Campania Luigi Vanvitelli, Naples, Italy. ${ }^{2}$ Ph.D. of Translational Medicine, Chair of Endocrinology and Metabolic Diseases, Department of Advanced Medical and Surgical Sciences, University of Campania Luigi Vanvitelli, Naples, Italy. ${ }^{3}$ Diabetes Unit, Department of Advanced Medical and Surgical Sciences, University of Campania Luigi Vanvitelli, Naples, Italy.

Received: 27 September 2021 Accepted: 7 October 2021

Published online: 12 October 2021

\section{References}

1. Grundy SM, Benjamin IJ, Burke GL, et al. Diabetes and cardiovascular disease: a statement for healthcare professionals from the American Heart Association. Circulation. 1999;100:1134-46.

2. Einarson TR, Acs A, Ludwig C, Panton UH. Prevalence of cardiovascular disease in type 2 diabetes: a systematic literature review of scientific evidence from across the world in 2007-2017. Cardiovasc Diabetol. 2018;17:83.

3. Bragg F, Holmes MV, lona A, et al. Association between diabetes and cause-specific mortality in rural and urban areas of China. JAMA. 2017;317:280-9.

4. Rawshani A, Rawshani A, Franzén S, et al. Risk factors, mortality, and cardiovascular outcomes in patients with type 2 diabetes. N Engl J Med. 2018:379:633-44.

5. Wright AK, Suarez-Ortegon MF, Read SH, et al. Risk factor control and cardiovascular event risk in people with type 2 diabetes in primary and secondary prevention settings. Circulation. 2020;142:1925-36.

6. Cavallari I, Bhatt DL, Steg G, et al. Causes and risk factors for death in diabetes: a competing-risk analysis from the SAVOR-TIMI 53 trial. JACC. 2021;77:1837-40

7. Giugliano D, Scappaticcio L, Longo M, et al. GLP-1 receptor agonists and cardiorenal outcomes in type 2 diabetes: an updated meta-analysis of eight CVOTs. Cardiovasc Diabetol. 2021;20:189.

8. Sattar N, Lee MMY, Kristensen SL, et al. Cardiovascular, mortality, and kidney outcomes with GLP-1 receptor agonists in patients with type 2 diabetes: a systematic review and meta-analysis of randomised trials. Lancet Diabetes Endocrinol. 2021;9:653-62.

9. American Diabetes Association. Pharmacologic approaches to glycemic treatment: standards of medical care in diabetes. Diabetes Care. 2021;44(Supplement 1):111-24.

10. Giugliano D, Longo M, Caruso P, Maiorino MI, Bellastella G, Esposito K. Sodium-glucose co-transporter-2 inhibitors for the prevention of cardiorenal outcomes in type 2 diabetes: an updated meta-analysis. Diabetes Obes Metab. 2021;23:1672-6.

11. Salah HM, Al'Aref SJ, Khan MS, et al. Effect of sodium-glucose cotransporter 2 inhibitors on cardiovascular and kidney outcomes - systematic review and meta-analysis of randomized placebo-controlled trials. Am Heart J. 2021;232:10-22.

12. Anker SD, Butler J, Filippatos G, et al. Empagliflozin in heart failure with a preserved ejection fraction. N Engl J Med. DOl: https://doi.org/10.1056/ NEJMoa2107038.

13. McMurray JJV, Solomon SD, Inzucchi SE, et al. Dapagliflozin in patients with heart failure and reduced ejection fraction. N Engl J Med. 2019;381:1995-2008.

14. Packer M, Anker SD, Butler J, et al. Cardiovascular and renal outcomes with empagliflozin in heart failure. N Engl J Med. 2020;383:1413-24

15. Fei Y, Tsoi MF, Cheung BMY. Cardiovascular outcomes in trials of new antidiabetic drug classes: a network meta-analysis. Cardiovasc Diabetol. 2019;18:112.

16. Ferro EG, Elshazly MB, Bhatt DL. New antidiabetes medications and their cardiovascular and renal benefits. Cardiol Clin. 2021;39:335-51.

17. D'Onofrio L, Mignogna C, Carlone A, et al. Decrease of coronary heart disease risk with GLP1-receptor agonists or SGLT2 inhibitors therapy in patients with type 2 diabetes in primary cardiovascular prevention: a 24 months follow-up study. Diabetes Res Clin Pract. 2021;173:108681.

18. DeRemer CE, Vouri SM, Guo J, Donahoo WT, Winterstein AG, Shao H. Comparing cardiovascular benefits between GLP-1 receptor agonists and SGLT2 inhibitors as an add-on to metformin among patients with type 2 diabetes: a retrospective cohort study. Journal Diabetes Complications. 2021;35:107972.

19. Schernthaner G, Shehadeh N, Ametov AS, et al. Worldwide inertia to the use of cardiorenal protective glucose-lowering drugs (SGLT2i and GLP-1RA) in high-risk patients with type 2 diabetes. Cardiovasc Diabetol. 2020;19:185.

20. Giugliano D, Maiorino MI, Bellastella G, Esposito K. Clinical inertia, reverse clinical inertia, and medication non-adherence in type 2 diabetes. J Endocrinol Invest. 2019;42:495-503.

21. Marx N, Davies MJ, Grant PJ, et al. Guideline recommendations and the positioning of newer drugs in type 2 diabetes care. Lancet Diabetes Endocrinol. 2021;9:46-52. 
22. Fang $M$, Wang $D$, Coresh J, Selvin E. Trends in diabetes treatment and control in U.S. adults, 1999-2018. N Engl J Med. 2021;384:2219-28.

23. Gregg EW, Hora I, Benoit SR. Resurgence in diabetes-related complications. JAMA. 2019;321:1867-8.

24. Rangaswami J, Bhalla V, de Boer IH, et al. Cardiorenal protection with the newer antidiabetic agents in patients with diabetes and chronic kidney disease: a scientific statement from the American Heart Association. Circulation. 2020;142:e265-86.

\section{Publisher's Note}

Springer Nature remains neutral with regard to jurisdictional claims in published maps and institutional affiliations.
Ready to submit your research? Choose BMC and benefit from:

- fast, convenient online submission

- thorough peer review by experienced researchers in your field

- rapid publication on acceptance

- support for research data, including large and complex data types

- gold Open Access which fosters wider collaboration and increased citations

- maximum visibility for your research: over $100 \mathrm{M}$ website views per year

At BMC, research is always in progress.

Learn more biomedcentral.com/submissions 